\title{
Error Analysis in Measured Conductivity under Low Induction Number Approximation for Electromagnetic Methods
}

\author{
George Caminha-Maciel $^{1,2}$ and Irineu Figueiredo ${ }^{1,3}$ \\ ${ }^{1}$ Observatório Nacional (ON), MCT, Rua General José Cristino 77, 20921-400 Rio de Janeiro, RJ, Brazil \\ ${ }^{2}$ Universidade Federal do Pampa (UNIPAMPA), Avenida Pedro Anunciação s/n', 96570-000 Caçapava do Sul, RS, Brazil \\ ${ }^{3}$ Universidade Estadual do Rio de Janeiro (UERJ), Rua São Francisco Xavier 524, 20550-900 Rio de Janeiro, RJ, Brazil
}

Correspondence should be addressed to George Caminha-Maciel; caminha.maciel@unipampa.edu.br

Received 28 August 2013; Accepted 5 November 2013

Academic Editors: G. Chisham, A. De Santis, G. Mele, and A. Tzanis

Copyright (C) 2013 G. Caminha-Maciel and I. Figueiredo. This is an open access article distributed under the Creative Commons Attribution License, which permits unrestricted use, distribution, and reproduction in any medium, provided the original work is properly cited.

\begin{abstract}
We present an analysis of the error involved in the so-called low induction number approximation in the electromagnetic methods. In particular, we focus on the EM34 equipment settings and field configurations, widely used for geophysical prospecting of laterally electrical conductivity anomalies and shallow targets. We show the theoretical error for the conductivity in both vertical and horizontal dipole coil configurations within the low induction number regime and up to the maximum measuring limit of the equipment. A linear relationship may be adjusted until slightly beyond the point where the conductivity limit for low induction number $(B=1)$ is reached. The equations for the linear fit of the relative error in the low induction number regime are also given.
\end{abstract}

\section{Introduction}

The induction method consists basically in determining subsurface rock conductivities with the help of electromagnetic fields generated by a coil at the Earth's surface and by catching the response to this field from the conducting media under surface by using a reception coil [1-3].

From the Maxwell equations, in particular the Faraday induction law applied to an infinite homogenous half-plane, the subsurface rock conductivity can be estimated through the ratio between the magnetic field measured in the receiving coil and the magnetic field produced at the transmission coil with both at surface. Then we can take laterally distributed measurements along a transect for identifying conductivity-related anomalies. We can also get information on the vertical conductivity structure by varying the coil's dipole configurations (vertical dipole or horizontal dipole) as well as by increasing the instrument height. This information is very useful in several geophysical problems as, for example, water prospecting or mapping pollution plumes.
The basic model for both configurations is described in Figure 1 where a transmission coil Tx with a given alternate electric current at a given frequency is located on the terrain (assumed to be an uniform semiplane) and a receiving coil $\mathrm{Rx}$ is located at a short distance $s$ from Tx. The time variation of the magnetic field $H_{p}$, called primary magnetic field, produced by the electric current in the transmission coil generates a small alternate current in the soil. This electric current, on its turn, produces a magnetic field $H_{s}$, called secondary field, which can be measured at the receiving coil together with the primary field.

In general, the secondary magnetic field $H_{s}$ is a complicated function of the distance between coils $s$, of the magnetic permeability of the medium $\mu$-which will be considered exactly the same as for vacuum $\mu_{0}\left(4 \pi \cdot 10^{-7} \mathrm{~A} \cdot \mathrm{m}\right)$ - of the angular frequency of the oscillatory electric current $\omega$, and of the soil conductivity $\sigma$. Under well-established conditions, technically known as "operation with low values of induction number," the secondary magnetic field $H_{s}$ becomes a simple 


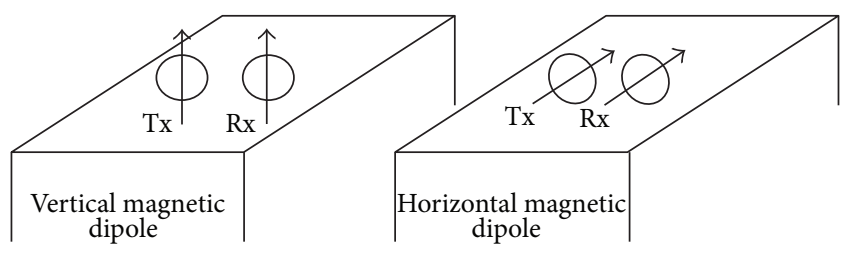

FIgURE 1: Representation of the transmission (Tx) and reception $(\mathrm{Rx})$ coils for both the vertical and the horizontal dipole configurations.

function of these variables when we consider only the quadrature component:

$$
\frac{H_{s}}{H_{p}} \cong \frac{\omega \mu \sigma}{4} s^{2} .
$$

This result can be used when coils are in the vertical or horizontal dipole orientations. Actually, these two situations are described by different sets of equations for the secondary field; however, these equations give the same result for the component in quadrature of the ratio between the primary and secondary fields under low induction number conditions.

In order to understand the induction number we need to define the "electromagnetic skin depth" $\delta$ :

$$
\delta=\left(\frac{2}{\omega \mu \sigma}\right)^{1 / 2},
$$

which is equal to the distance in a conducting medium an electromagnetic front wave has to travel in order to reduce the amplitude to $1 / e(\sim 37 \%)$ of its value outside the conducting medium.

The induction number $B$ describes the distance between the transmission coil and the reception coil $s$ in units of skin depth $\delta$ :

$$
B=\frac{s}{\delta}
$$

The recent literature contains some debate on which values of $B$ (and consequently $\sigma$ ) are valid for low induction number approximations $[4,5]$. It also contains suggested corrections for nonzero height dependence and nonlinear departure for higher values of conductivity even above the low induction number regime [4].

In this work we introduce an error analysis for the induction method taking as a starting point the set of equations given by McNeill [1] for the field ratios on both coil dipole configurations. We also consider zero elevation of the coils and, as in Beamish [4], we discard medium magnetic and dielectric components as we are working in frequencies lower than $15 \mathrm{kHz}$. Those equations represent the ratio between primary and secondary fields as the response of a homogeneous earth. We calculate the relative difference between this conductivity value and that obtained through the low induction number approximation.

\section{Methods}

The equations for the primary and secondary field ratios for vertical and horizontal dipole configurations as given by McNeill [1] are

$$
\begin{gathered}
\left(\frac{H_{s}}{H_{p}}\right)_{V} \\
=\frac{2}{(\gamma s)^{2}} \cdot\left\{9-\left\{9+9 \cdot(\gamma s)+4 \cdot(\gamma s)^{2}+(\gamma s)^{3}\right\} \cdot e^{-\gamma s}\right\}, \\
\left(\frac{H_{s}}{H_{p}}\right)_{H}=2 \cdot\left\{1-\frac{3}{(\gamma s)^{2}}+\left[3+3 \cdot(\gamma s)+(\gamma s)^{2}\right] \cdot \frac{e^{-\gamma s}}{(\gamma s)^{2}}\right\},
\end{gathered}
$$

where

$$
\begin{aligned}
& \gamma=\sqrt{\left(i \omega \mu_{0} \sigma\right)}, \\
& \omega=2 \pi f, \\
& f=\text { frequency }(\mathrm{Hz}), \\
& \mu_{0}=\text { permeability of free space, } \\
& i=\sqrt{-1} .
\end{aligned}
$$

Expressions (4) and (5) are multivalued functions of the conductivity, frequency, and intercoil spacing, and as functions of the complex exponential $\sqrt{i}$ they have several branches in the complex plane. Therefore, as a matter of simplification, the induction number, a nondimensional parameter defined as the ratio between intercoil spacing and skin depth, is introduced, which will act as a conformal map simplifying equations.

To do this we begin by writing $x=i \gamma s$ as a function of the induction number $B=s / \delta$ :

$$
x=\sqrt{\frac{2}{i}} B .
$$

Remembering that

$$
(1-i)^{2}=-2 i=\frac{2}{i}
$$

we have

$$
x=(1-i) B .
$$

By substituting in (4) the following expression for the vertical dipole configuration comes out:

$$
\begin{aligned}
&\left(\frac{H_{s}}{H_{p}}\right)_{V}= h_{V} \\
&=-\frac{18}{(1-i)^{2} B^{2}}\left[1-\left(1-(1-i) B+\frac{4}{9}(1-i)^{2} B^{2}\right.\right. \\
& \\
&\left.\left.\quad-\frac{1}{9}(1-i)^{3} B^{3}\right) e^{(1-i) B}\right] .
\end{aligned}
$$


As

$$
(1-i)^{2}=-2 i, \quad(1-i)^{3}=-2(1+i),
$$

then

$$
h_{V}=-\frac{9 i}{B^{2}}\left[1-\left(1-B+i B-\frac{8}{9} i B^{2}+\frac{2}{9} B^{3}+\frac{2}{9} i B^{3}\right) e^{B} e^{-i B}\right],
$$

and doing $e^{-i B}=\cos B-i \sin B$, it follows that

$$
\begin{aligned}
h_{V}=-\frac{1}{B^{2}}[9 i+ & {\left[\left(9 B-8 B^{2}+2 B^{3}\right)-\left(9-9 B+2 B^{3}\right) i\right] } \\
& \left.\times e^{B}[\cos B-i \sin B]\right] .
\end{aligned}
$$

By taking only the quadrature of $h$ we have

$$
\begin{aligned}
Q h_{V}=-\frac{1}{B^{2}}[9- & \left(9 B-8 B^{2}+2 B^{3}\right) e^{B} \sin B \\
- & \left.\left(9-9 B+2 B^{3}\right) e^{B} \cos B\right] .
\end{aligned}
$$
obtain

Similarly, for the horizontal dipole configuration we

$$
Q h_{H}=-\frac{1}{B^{2}}\left[-3+\left(3 B-2 B^{2}\right) e^{B} \sin B+(3-3 B) e^{B} \cos B\right] .
$$

The imaginary part (quadrature term) is related to the conductivity measurements under low induction number conditions. The magnitude of the secondary magnetic field is now directly proportional to the conductivity and its phase leads the primary field to $90^{\circ}$. Under low induction number $(B \ll 1)$ the expressions $(4)$ and $(5)$ can be expanded in a power series, and considering only the first terms the quadrature part (neglecting the in-phase term) can be written as [1]

$$
\left(\frac{H_{s}}{H_{p}}\right)_{V}=\left(\frac{H_{s}}{H_{p}}\right)_{H}=\frac{i \sigma \mu \omega}{4} s^{2} .
$$

This is the low induction number approximation key expression which leads to the conductivity value from the equipment readings.

The induction number $B$ is a function of the material conductivity $\sigma$ for a given frequency $\omega$ and the separation between coils $s$. Furthermore, $B$ is a function of the conductivity $\sigma$ and of the product $\left(f^{1 / 2} * s\right)$. For the EM34-3 and EM34-X (Geonics Ltd.) equipment settings all different possibilities of frequency and intercoil spacing give the same product $f^{1 / 2} * s\left(800 \mathrm{mS}^{1 / 2} * \mathrm{~m}^{1 / 2}\right)$. Since the low induction number condition $(B \ll 1)$ implies that we should have $\sigma \ll$ $2 / \mu \omega s^{2}$, then, considering the EM34-3 instrument that works in the frequencies 6400,1600 , and $400 \mathrm{~Hz}$ with distances between coils of 10,20 , and $40 \mathrm{~m}$, respectively, it comes out that the value for the soil conductivity measured by this instrument should be quite lower than $400 \mathrm{mS} / \mathrm{m}$.
We define the error associated with the low induction number approximation as the theoretical deviation from the exact model which is the electromagnetic response for a homogenous half-plane as follows:

$$
\varepsilon=\frac{\left|\sigma^{\prime}-\sigma\right|}{\sigma} .
$$

From the induction number definition we have

$$
\sigma=\frac{2 B^{2}}{\mu \omega s^{2}},
$$

and from (15)

$$
\sigma^{\prime}=\frac{4(Q h)}{\mu \omega s^{2}} .
$$

In this way, the error associated with the approximation is

$$
\varepsilon=\frac{\left|4(Q h)-2 B^{2}\right|}{2 B^{2}}=\left|\frac{2(Q h)}{B^{2}}-1\right| .
$$

Substituting those $Q h$ values in expression (19) we obtain the relative theoretical error for the approximation. Figure 2 represents the plot of the error for the whole conductivity range (and a little further) mostly used in practical applications $\left(10^{-2} \mathrm{mS} / \mathrm{m}-10^{3} \mathrm{mS} / \mathrm{m}\right)$ and shows the deviation from the linear response. We add two vertical black lines marking the limits of interest: the low induction number limit $(B=1)$ and the usual limit of the equipment $(1000 \mathrm{mS} / \mathrm{m})$.

From the studied lower limit through the whole low induction number regime (considering its limit as 400/10 = $40 \mathrm{mS} / \mathrm{m}$ ) the relationship is clearly linear, but it also can easily be extended to $100 \mathrm{mS} / \mathrm{m}$ or even to $400(\mathrm{mS}) / \mathrm{m}(B=$ 1 ), as it is shown in Figure 2.

Figure 3 shows the absolute apparent conductivity as a function of true conductivity until the linear response limit is surpassed. McNeill [1] shows this plot for a narrower range of conductivity values, and therefore the departure from linearity was not clear. The same linear behaviour is seen in the apparent conductivity (Figure 3) for vertical and horizontal dipole configurations, as seen by the fitted straight line to the logarithmic scaled error curves on the low induction number regime $\left(10^{-2} \mathrm{mS} / \mathrm{m}-40 \mathrm{mS} / \mathrm{m}\right)$ given by

$$
\begin{aligned}
& \log (\varepsilon) \\
& =\left\{\begin{array}{c}
0.4915 \cdot \log \left(\sigma_{V}\right)+0.7321 \longrightarrow \text { Vertical dipole } \\
0.4959 \cdot \log \left(\sigma_{H}\right)+0.4296 \longrightarrow \text { Horizontal dipole }
\end{array}\right\},
\end{aligned}
$$

where $\varepsilon$ is given in percent (\%) and $\sigma$ in $\mathrm{mS} / \mathrm{m}$.

\section{Conclusions}

It is well known that the vertical dipole configuration is more sensitive to conductivity anomalies in deep, while the horizontal one is more sensitive to near surface conductivity 


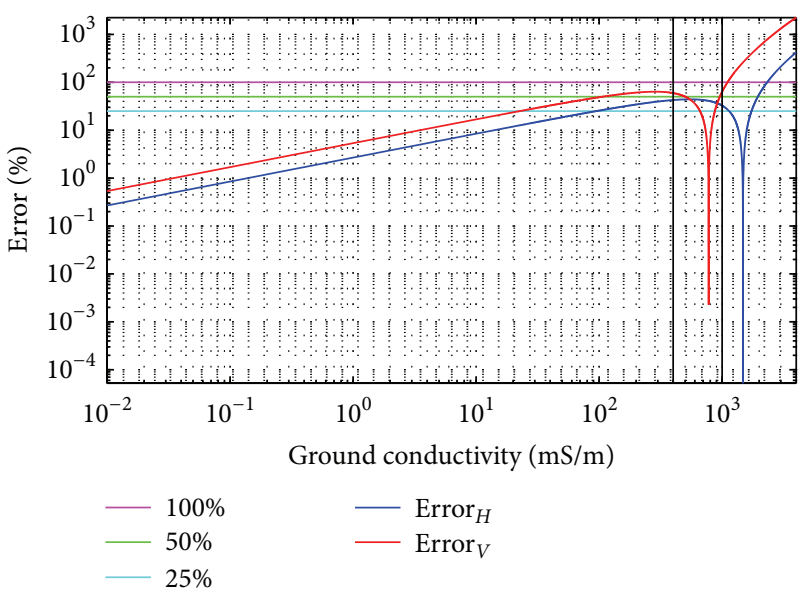

FIGURE 2: Relative percent error (\%) as a function of conductivity $(\mathrm{mS} / \mathrm{m})$. It also shows error isolines for the error levels of $100 \%, 50 \%$, and $25 \%$. The vertical black lines mark two main limits: induction number equals to one ( $B=1$, for which $\sigma \sim 400 \mathrm{mS} / \mathrm{m})$ and the EM34 instrument limit of $1000 \mathrm{mS} / \mathrm{m}$.

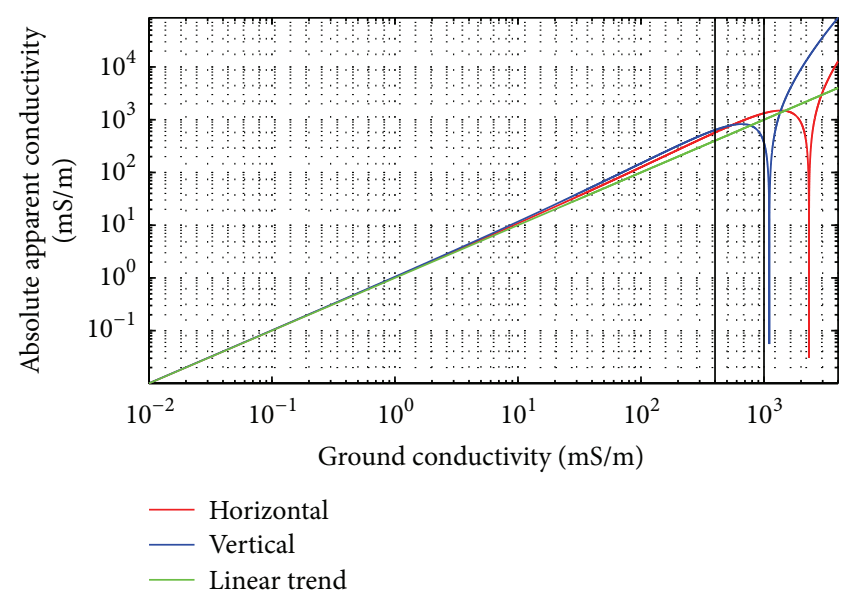

FIGURE 3: Apparent conductivity versus true conductivity in $\mathrm{mS} / \mathrm{m}$ until slightly above the EM34 instrument limit. The vertical black lines mark two main limits: induction number equals to one $(P=$ 1 , for which $\sigma \sim 400 \mathrm{mS} / \mathrm{m}$ ) and the EM34 instrument limit of $1000 \mathrm{mS} / \mathrm{m}$. This curve shows a slight departure from the linear behaviour after the low induction number limit $(\sim 40 \mathrm{mS} / \mathrm{m})$ until the limit for $B=1(\sim 400 \mathrm{mS} / \mathrm{m})$ and a strong departure from the linear behaviour after that.

variations [1]. However, if we consider only the measured conductivity values, the error is lower for the horizontal than for the vertical dipole configuration as noted in Figure 2. For both configurations conductivity converges to the same values when conductivity tends to zero.

An approximately linear tendency can be observed over the whole low induction number range and up to $400 \mathrm{mS} / \mathrm{m}$. Above this value there are strong deviations from linearity indicating that we are close to singularities of the complex function (not only the quadrature term). Despite a correction procedure suggested by Beamish [4], we believe that under statistical fluctuations of the measured signal the response could depart from the expected model leading to very high errors in the estimated conductivity due to the proximity of the function singularity.

It is worth noting that the determination of these bounds depends on the configurations (operation frequency and intercoil spacing) of the considered instrument.

\section{Acknowledgments}

This work was supported by the Brazilian agency $\mathrm{CNPq}$ through a postdoctoral scholarship to George CaminhaMaciel. Thanks are due to E. La Terra for the valuable discussions and M. Ernesto for helping in improving the paper. Any use of product names is for descriptive purposes only and does not imply any endorsement.

\section{References}

[1] J. D. McNeill, "Electromagnetic terrain conductivity measurements at low induction numbers," Technical Note TN-6, Geonics Ltd., Mississauga, Canada, 1980.

[2] G. V. Keller and F. C. Firschknecht, Electrical Methods in Geophysical Prospecting, Pergamon, New York, NY, USA, 1966.

[3] J. R. Waitt, "A note on the electromagnetic response of a stratified Earth," Geophysics, vol. 27, pp. 382-385, 1962.

[4] D. Beamish, "Low induction number, ground conductivity meters: A correction procedure in the absence of magnetic effects," Journal of Applied Geophysics, vol. 75, no. 2, pp. 244-253, 2011.

[5] J. B. Callegary, T. P. A. Ferré, and R. W. Groom, "Vertical spatial sensitivity and exploration depth of low-induction-number electromagnetic-induction instruments," Vadose Zone Journal, vol. 6, no. 1, pp. 158-167, 2007. 

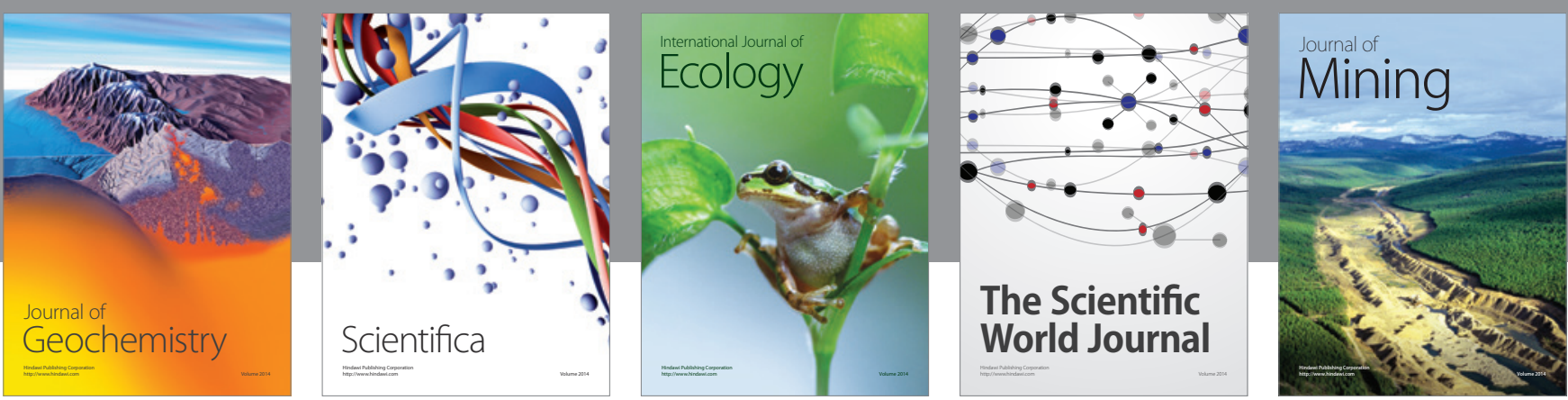

The Scientific World Journal
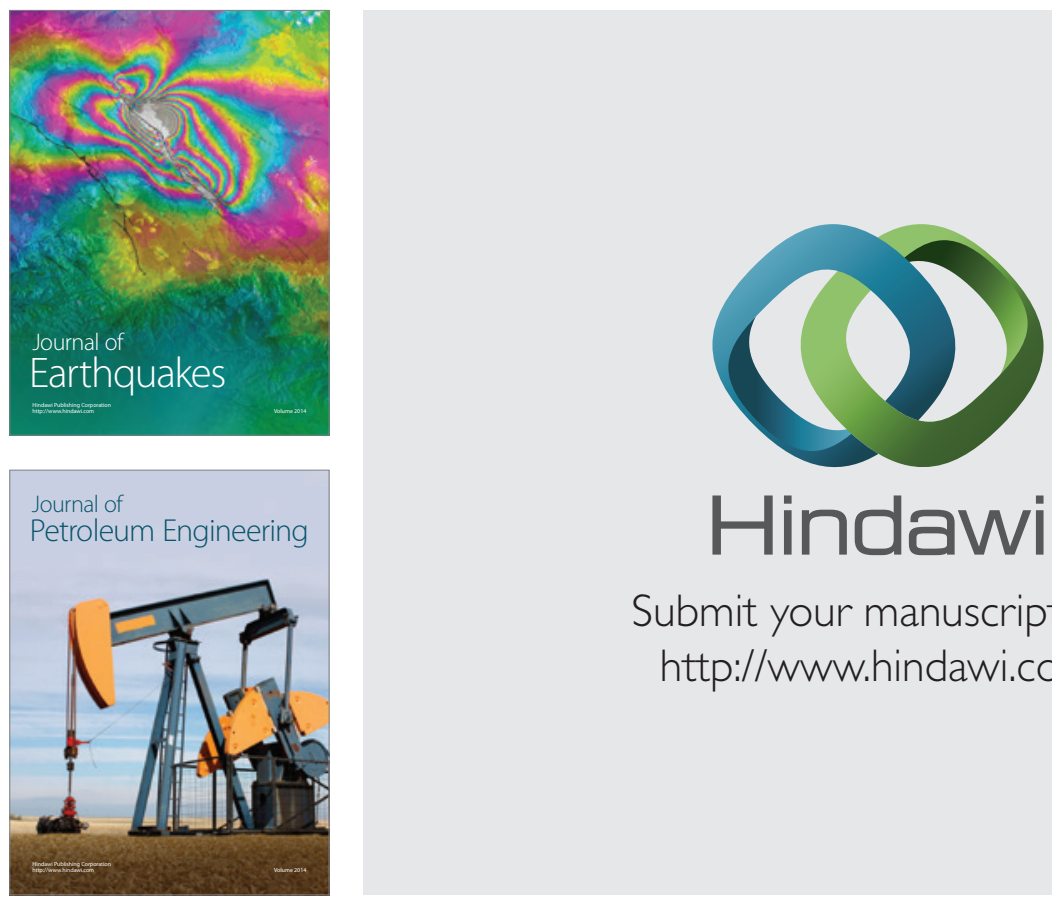

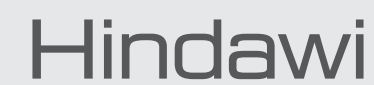

Submit your manuscripts at

http://www.hindawi.com
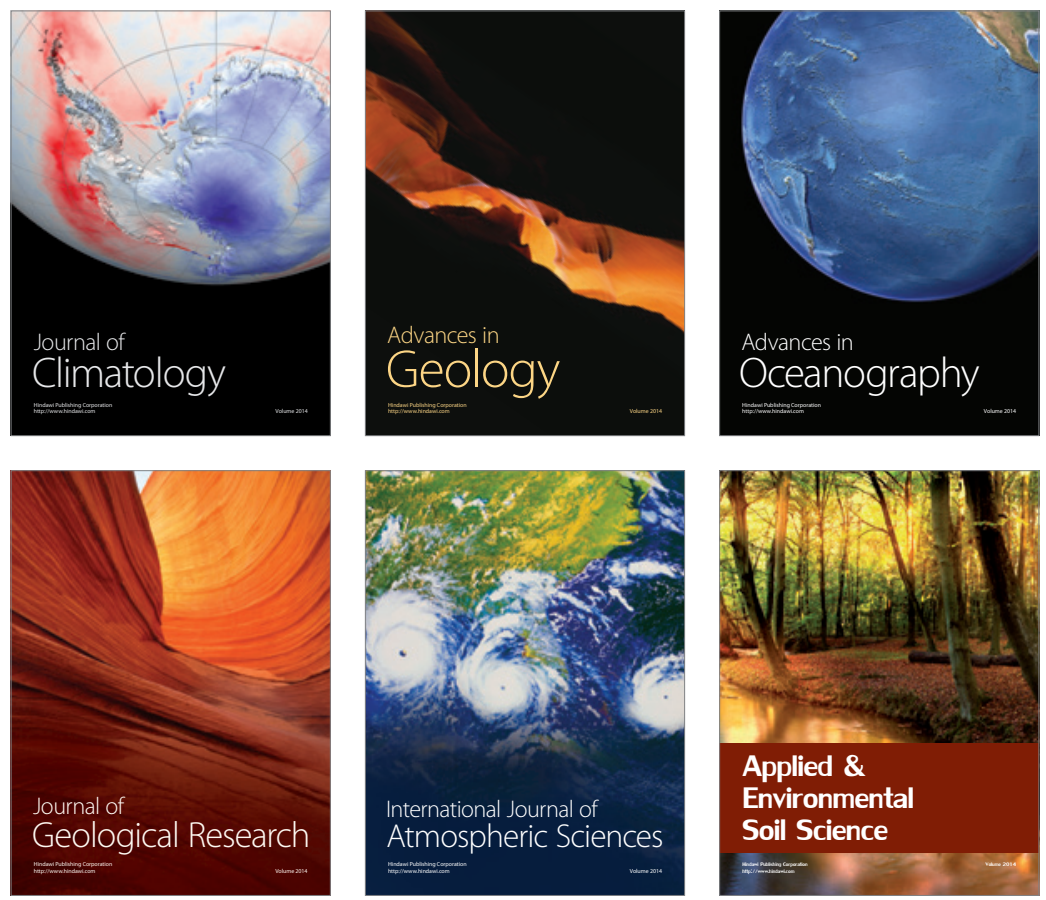
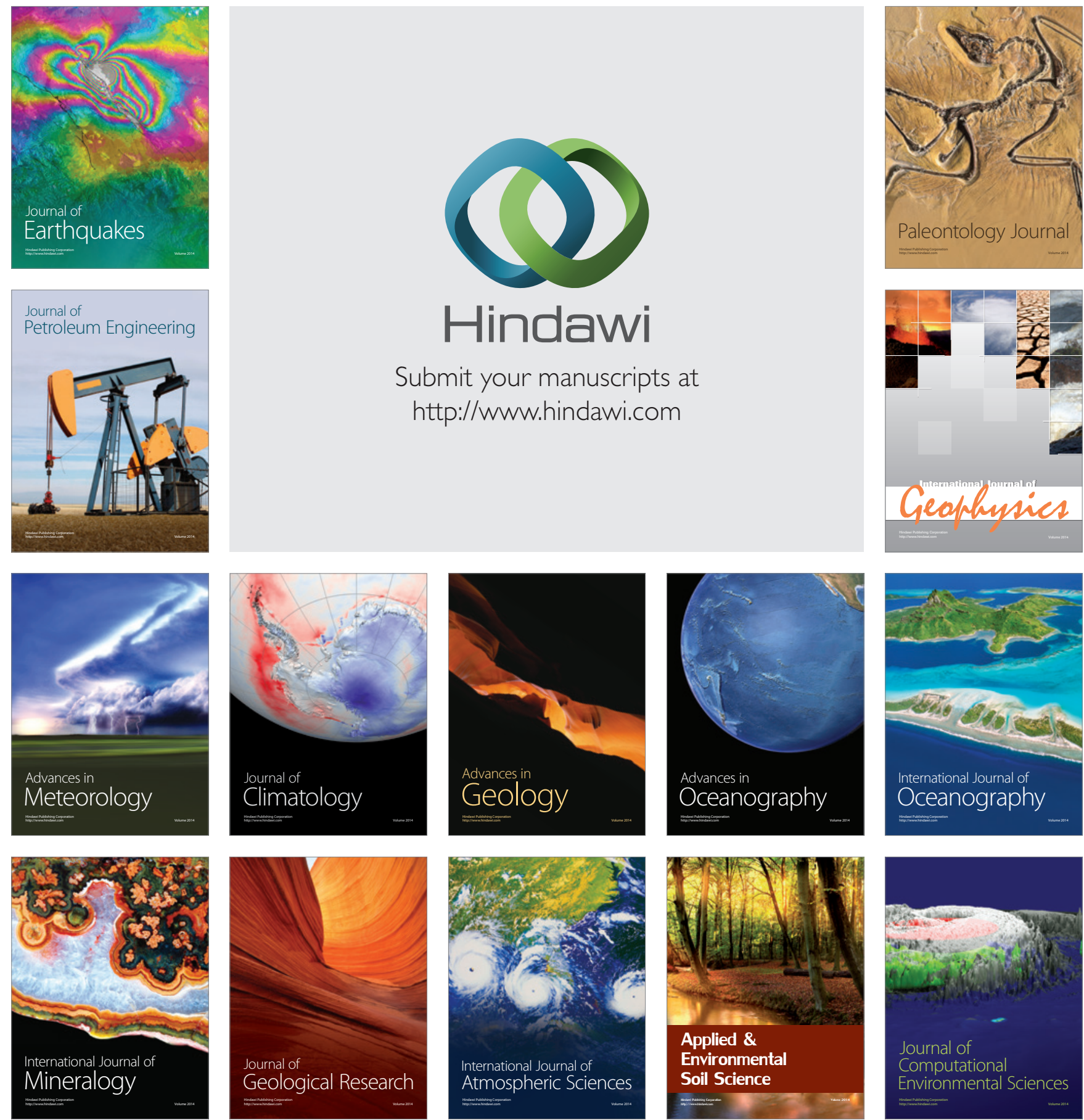